\title{
A CELEBRATION OF JÜRG AND TOM
}

\author{
BARRY SIMON*
}

It is both a pleasure and an honor to write the introduction of this issue in honor of the (recent) sixtieth birthdays of Jürg Fröhlich and Tom Spencer and to be able to place their joint work in some perspective.

Tom and Jürg have about twenty-five joint papers, several with additional authors including two with me. I want to focus here on two sets of methods: infrared bounds and multiscale analysis, which are surely among the most significant developments in rigorous statistical physics in the last quarter of the last century.

Infrared bounds $([29,30])$, discovered in 1975 and proven using reflection positivity, provide upper bounds on the Fourier transform of the spin-spin correlation at nonzero momentum and force a macroscopic occupation of zero momentum at low temperature (aka Bose-Einstein condensation of spin waves). This implies long-range order, and so, a phase transition.

The method was used for quantum spin antiferromagnets by DysonLieb-Simon [20, 21]. Remarkably, after more than thirty years, it remains the only method known to rigorously prove breaking of nonabelian symmetry - even for the abelian case, there is only one other approach to the short-range case using multiscale analysis (see below). For slow decay two-dimensional plane rotors, there are also results of Kunz-Pfister [49].

Among later applications of infrared bounds are Sokal's specific heat bounds [59], Aizenman's [1] and Fröhlich's [24] proofs of the triviality of $\phi^{4}$ theories in five or more dimensions, the Aizenman-Fernández analysis of long-range models [4], Helffer's estimates of eigenvalue splitting for certain Schrödinger operators in the thermodynamic limit [43, 44], and the work of Biskup-Chayes on mean-field driven phase transitions $[7,8]$.

Reflection positivity was introduced in Euclidean field theory by Osterwalder-Schrader [54] and was a key element, albeit implicitly,

Date: September 15, 2008.

Mathematics 253-37, California Institute of Technology, Pasadena, CA 91125. E-mail: bsimon@caltech.edu. Supported in part by NSF grant DMS-0652919. 
in the work of FSS [29, 30]. This was a partial motivation for a series of works on chessboard estimates, many on phase transitions; for example, $[9,10,11,17,22,25,26,27,50,51,52]$.

This material is further discussed in the review article of Shlosman [55] and in four books [42, 39, 23, 57].

We turn next to multiscale analysis. It is, of course, ancient wisdom to study infinite volume systems by approximating by finite volumes, say, cubes of length $L$. Before the Fröhlich-Spencer breakthrough, the sequence of $L$ 's one looks at were typically $L_{k+1}=L_{k}+1$, or if one were especially brave, $L_{k+1}=2 L_{k}$. Multiscale analysis looks at $L_{k+1}=\left(L_{k}\right)^{\alpha}$ for some $\alpha>1$.

At any level, there are typically good boxes where one gets estimates of a convenient form and bad boxes where estimates are much weaker. One decomposes $L_{k+1}$ boxes into $\left(L_{k}\right)^{\nu(\alpha-1)}$ ( $\nu=$ dimension) boxes of side $L_{k}$. If most of these smaller boxes are good, $L_{k+1}$ is good. In this way, one inductively gets estimates on good boxes, proving that as $k \rightarrow \infty$, all boxes but a vanishingly small number are good.

What is particularly fascinating about the situation is that the underlying physics is either scaleless or has a single scale, but the mathematical machinery uses these multiple scales.

The breakthrough appeared first in 1981 in their proof of the Kosterlitz-Thouless transition [31, 32] and was later used by Jürg and Tom to analyze the phase transition in the one-dimensional Ising model with $1 / r^{2}$ interaction energy [33], the deconfinement transition in fourdimensional $U(1)$ gauge theory [34], the phase transition in the threeor higher-dimensional plane rotor model (obtained already by FSS) [35], and localized states for certain quasi-periodic Schrödinger operators describing a particle hopping on the integers or moving on the real line [38]. Of course, these papers also used other clever ideas and techniques. Among precursors, one should certainly single out the paper of Glimm-Jaffe [41].

With Wayne [37], Jürg and Tom applied multiscale analysis to construct invariant tori in some Hamiltonian systems with infinitely many degrees of freedom; for later work in this direction, see [12, 13]. Except for localization, most of the results obtained by multiscale analysis have not been obtained by other methods. For localization, there is another approach found ten years later: the fractional moment method of Aizenman-Molchanov [5, 2, 3, 6]. 
In [36], Fröhlich and Spencer proved exponential decay of the Green's function in the spectrum for the Anderson model and left open the expected existence of point spectrum with exponentially decaying eigenfunctions. This was supplied by [28]. Shortly thereafter, the now standard way of going from Green's function decay to point spectrum was found by Simon-Wolff [56]. Further important localization criteria go under the names of dynamical localization and semi-uniform localization of eigenfunctions (SULE) - these can be tracked down through the reviews mentioned below.

Critical reworkings of multiscale analysis were developed by von Dreifus in his thesis [18] and with Klein [19]. Among extensions to settings beyond the lattice models that [36] consider, I would mention $[14,15,16,40,46,48,53]$; see the review articles mentioned next for more.

For book or book-length presentations of multiscale analysis for the Anderson model, see Stollmann [58] and Kirsch [45]. For a comprehensive review, see Klein [47].

Jürg and Tom: you have provided the seed-corn for a generation of mathematical physicists. So thanks and many happy returns.

\section{REFERENCES}

[1] M. Aizenman, Geometric analysis of $\phi^{4}$ fields and Ising models, I, II, Comm. Math. Phys. 86 (1982), 1-48.

[2] M. Aizenman, Localization at weak disorder: some elementary bounds, Rev. Math. Phys. 6 (1994), 1163-1182.

[3] M. Aizenman, A. Elgart, S. Naboko, J. H. Schenker, and G. Stolz, Moment analysis for localization in random Schrödinger operators, Invent. Math. 163 (2006), 343-413.

[4] M. Aizenman and R. Fernández, Critical exponents for long-range interactions, Lett. Math. Phys. 16 (1988), 39-49.

[5] M. Aizenman and S. Molchanov, Localization at large disorder and at extreme energies: an elementary derivation, Comm. Math. Phys. 157 (1993), 245-278.

[6] M. Aizenman, J. H. Schenker, R. M. Friedrich, and D. Hundertmark, Finitevolume fractional-moment criteria for Anderson localization, Comm. Math. Phys. 224 (2001), 219-253.

[7] M. Biskup and L. Chayes, Rigorous analysis of discontinuous phase transitions via mean-field bounds, Comm. Math. Phys. 238 (2003), 53-93.

[8] M. Biskup, L. Chayes, and N. Crawford, Mean-field driven first-order phase transitions in systems with long-range interactions, J. Statist. Phys. 122 (2006), 1139-1193.

[9] M. Biskup, L. Chayes, and S. A. Kivelson, Order by disorder, without order, in a two-dimensional spin system with $\mathrm{O}(2)$ symmetry, Ann. Henri Poincaré 5 (2004), 1181-1205. 
[10] M. Biskup, L. Chayes, and Z. Nussinov, Orbital ordering in transition-metal compounds, I. The 120-degree model, Comm. Math. Phys. 255 (2005), 253292.

[11] M. Biskup, L. Chayes, and S. Starr, Quantum spin systems at positive temperature, Comm. Math. Phys. 269 (2007), 611-657.

[12] J. Bourgain, Construction of quasi-periodic solutions for Hamiltonian perturbations of linear equations and applications to nonlinear PDE, Internat. Math. Res. Notices 1994, no. 11 (electronic).

[13] J. Bourgain, Quasi-periodic solutions of Hamiltonian perturbations of 2D linear Schrödinger equations, Ann. of Math. (2) 148 (1998), 363-439.

[14] J. Bourgain and C. E. Kenig, On localization in the continuous AndersonBernoulli model in higher dimension, Invent. Math. 161 (2005), 389-426.

[15] R. Carmona, A. Klein, and F. Martinelli, Anderson localization for Bernoulli and other singular potentials, Comm. Math. Phys. 108 (1987), 41-66.

[16] J.-M. Combes and P. D. Hislop, Localization for some continuous, random Hamiltonians in d-dimensions, J. Funct. Anal. 124 (1994), 149-180.

[17] R. L. Dobrushin and S. Shlosman, Phases corresponding to the local minima of the energy, Selecta Math. Soviet. 1 (1981), 317-338.

[18] H. von Dreifus, On the Effects of Randomness in Ferromagnetic Models and Schrödinger Operators, Ph.D. thesis, New York University, 1987.

[19] H. von Dreifus and A. Klein, A new proof of localization in the Anderson tight binding model, Comm. Math. Phys. 124 (1989), 285-299.

[20] F. J. Dyson, E. Lieb, and B. Simon, Phase transitions in the quantum Heisenberg model, Phys. Rev. Lett. 37 (1976), 120-123.

[21] F. J. Dyson, E. Lieb, and B. Simon, Phase transitions in quantum spin systems with isotropic and non-isotropic interactions, J. Statist. Phys. 18 (1978), $335-383$.

[22] A. C. D. van Enter and S. B. Shlosman, Provable first-order transitions for liquid crystal and lattice gauge models with continuous symmetries, Comm. Math. Phys. 255 (2005), 21-32.

[23] R. Fernández, J. Fröhlich, and A. Sokal, Random Walks, Critical Phenomena, and Triviality in Quantum Field Theory, Springer, Berlin, 1992.

[24] J. Fröhlich, On the triviality of $\lambda \varphi_{d}^{4}$ theories and the approach to the critical point in $d_{(-)}>4$ dimensions, Nuclear Phys. B 200 (1982), 281-296.

[25] J. Fröhlich, R. Israel, E. H. Lieb, and B. Simon, Phase transitions and reflection positivity, I. General theory and long range lattice models, Comm. Math. Phys. 62 (1978), 1-34.

[26] J. Fröhlich, R. Israel, E. H. Lieb, and B. Simon, Phase transitions and reflection positivity, II. Lattice systems with short-range and Coulomb interactions, J. Statist. Phys. 22 (1980), 297-347.

[27] J. Fröhlich and E. H. Lieb, Phase transitions in anisotropic lattice spin systems, Comm. Math. Phys. 60 (1978), 33-267.

[28] J. Fröhlich, F. Martinelli, E. Scoppola, and T. Spencer, Constructive proof of localization in the Anderson tight binding model, Comm. Math. Phys. 101 (1985), 21-46.

[29] J. Fröhlich, B. Simon, and T. Spencer, Phase transitions and continuous symmetry breaking, Phys. Rev. Lett. 36 (1976), 804-806. 
[30] J. Fröhlich, B. Simon, and T. Spencer, Infrared bounds, phase transitions and continuous symmetry breaking, Comm. Math. Phys. 50 (1976), 79-85.

[31] J. Fröhlich and T. Spencer, Kosterlitz-Thouless transition in the twodimensional plane rotator and Coulomb gas, Phys. Rev. Lett. 46 (1981), 1006-1009.

[32] J. Fröhlich and T. Spencer, The Kosterlitz-Thouless transition in twodimensional abelian spin systems and the Coulomb gas, Comm. Math. Phys. 81 (1981), 527-602.

[33] J. Fröhlich and T. Spencer, The phase transition in the one-dimensional Ising model with $1 / r^{2}$ interaction energy, Comm. Math. Phys. 84 (1982), 87-101.

[34] J. Fröhlich and T. Spencer, Massless phases and symmetry restoration in abelian gauge theories and spin systems, Comm. Math. Phys. 83 (1982), 411454.

[35] J. Fröhlich and T. Spencer, The Berezinskii-Kosterlitz-Thouless transition, in "Scaling and Self-Similarity in Physics: Renormalization in Statistical Mechanics and Dynamics (J. Fröhlich, ed.)," Progress in Physics, Birkhäuser, Basel-Boston, 1983.

[36] J. Fröhlich and T. Spencer, Absence of diffusion in the Anderson tight binding model for large disorder or low energy, Comm. Math. Phys. 88 (1983), 151184.

[37] J. Fröhlich, T. Spencer, and C. E. Wayne, Localization in disordered, nonlinear dynamical systems, J. Statist. Phys. 42 (1986), 247-274.

[38] J. Fröhlich, T. Spencer, and P. Wittwer, Localization for a class of onedimensional quasi-periodic Schrödinger operators, Comm. Math. Phys. 132 (1990), 5-25.

[39] H. O. Georgii, Gibbs Measures and Phase Transitions, De Gruyter Studies in Mathematics 9, de Gruyter, Berlin, 1988.

[40] F. Germinet, P. D. Hislop, and A. Klein, Localization for Schrödinger operators with Poisson random potential, J. Eur. Math. Soc. (JEMS) 9 (2007), 577-607.

[41] J. Glimm and A. Jaffe, Positivity of the $\phi_{3}^{4}$ Hamiltonian, Fortschr. Physik 21 (1973), 327-376.

[42] J. Glimm and A. Jaffe, Quantum Physics - A Functional Integral Point of View, 2nd ed., Springer-Verlag, Heidelberg, 1987.

[43] B. Helffer, Splitting in large dimension and infrared estimates, Microlocal Analysis and Spectral Theory (Lucca, 1996), pp. 307-347, NATO Adv. Sci. Inst. Ser. C: Math. Phys. Sci. 490, Kluwer, Dordrecht, 1997.

[44] B. Helffer, Splitting in large dimension and infrared estimates, II. Moment inequalities, J. Math. Phys. 39 (1998), 760-776.

[45] W. Kirsch, An invitation to random Schrödinger operators, to appear in "Panoramas et Synthèses," Soc. Math. de France.

[46] A. Klein, Extended states in the Anderson model on the Bethe lattice, Adv. Math. 133 (1998), 163-184.

[47] A. Klein, Multiscale analysis and localization of random operators, Panoramas et Synthèses 25 (2008), 1-39.

[48] F. Klopp, Localisation pour des opérateurs de Schrödinger aléatoires dans $L^{2}\left(\mathbb{R}^{d}\right)$ : un modéle semi-classique, Ann. Inst. Fourier (Grenoble) 45 (1995), 265-316. 
[49] H. Kunz and C.-E. Pfister, First order phase transition in the plane rotator ferromagnetic model in two dimensions, Comm. Math. Phys. 46 (1976), 245251.

[50] E. H. Lieb, Two theorems on the Hubbard model, Phys. Rev. Lett. 62 (1989), 1201-1204.

[51] E. H. Lieb, The flux phase of the half-filled band, Phys. Rev. Lett. 73 (1994), $2158-2161$.

[52] N. Macris and B. Nachtergaele, On the flux phase conjecture at half-filling: An improved proof, J. Statist. Phys. 85 (1996), 745-761.

[53] F. Martinelli and H. Holden, On absence of diffusion near the bottom of the spectrum for a random Schrödinger operator on $L^{2}\left(\mathbb{R}^{\nu}\right)$, Comm. Math. Phys. 93 (1984), 197-217.

[54] K. Osterwalder and R. Schrader, Axioms for Euclidean Green's functions, Comm. Math. Phys. 31 (1973), 83-112.

[55] S. Shlosman, The method of reflection positivity in the mathematical theory of the first-order phase transitions, Russian Math. Surveys 41 (1986), 83-134.

[56] B. Simon and T. Wolff, Singular continuous spectrum under rank one perturbations and localization for random Hamiltonians, Commun. Pure Appl. Math. 39 (1986), 75-90.

[57] Ya. G. Sinaŭ, Theory of Phase Transitions: Rigorous Results, International Series in Natural Philosophy 108, Pergamon Press, Oxford-Elmsford, N.Y., 1982.

[58] P. Stollmann, Caught by Disorder. Bound States in Random Media, Progress in Mathematical Physics 20, Birkhäuser, Boston, 2001.

[59] A. D. Sokal, A rigorous inequality for the specific heat of an Ising or $\phi^{4}$ ferromagnet, Phys. Lett. A 71 (1979), 451-453. 